\title{
A General Approach Towards Triazole-Linked Adenosine Diphosphate Ribosylated Peptides and Proteins
}

\author{
Qiang Liu, Hans A. V. Kistemaker, Sagar Bhogaraju, Ivan Dikic, Herman S. Overkleeft, \\ Gijsbert A. van der Marel, Huib Ovaa, Gerbrand J. van der Heden van Noort,* and
}

\section{Dmitri V. Filippov**}

\begin{abstract}
Current methods to prepare adenosine diphosphate ribosylated $(A D P r)$ peptides are not generally applicable due to the labile nature of this post-translational modification and its incompatibility with strong acidic conditions used in standard solid-phase peptide synthesis. A general strategy is presented to prepare ADPr peptide analogues based on a copper-catalyzed click reaction between an azide-modified peptide and an alkyne-modified ADPr counterpart. The scope of this approach was expanded to proteins by preparing two ubiquitin ADPr analogues carrying the biological relevant $\alpha$ glycosidic linkage. Biochemical validation using Legionella effector enzyme SdeA shows that clicked ubiquitin ADPr is well-tolerated and highlights the potential of this strategy to prepare ADPr proteins.
\end{abstract}

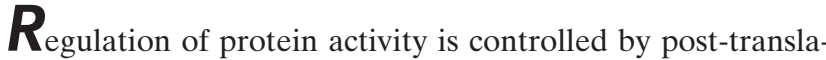
tional modifications (PTMs) that are installed on specific side-chain functionalities of amino acids in the involved protein. Simple PTMs, such as acetylation, methylation, and phosphorylation, have been subject of a large amount of studies, and the focus of PTM research is shifting to more complex PTMs. One of these PTMs is called adenosine diphosphate ribose (ADPr), a modification in which a specific nucleophilic side chain in the target protein displaces $\beta$ oriented nicotinamide from $\mathrm{NAD}^{+}$under the agency of an ADPr-transferase (ART) resulting in an $\alpha$-oriented glycosidic

[*] Q. Liu, Dr. H. A. V. Kistemaker, Prof. Dr. H. S. Overkleeft, Prof. Dr. G. A. van der Marel, Dr. D. V. Filippov

Bio-organic Synthesis, Leiden University

Einsteinweg 55, 2333 CC Leiden (The Netherlands)

E-mail: filippov@chem.leidenuniv.nl

Dr. S. Bhogaraju, Prof. Dr. I. Dikic

Institute of Biochemistry II, Goethe University Faculty of Medicine

Theodor-Stern-Kai 7, 60590 Frankfurt am Main (Germany)

and

Buchmann Institute for Molecular Life Sciences, Goethe University

Frankfurt, Riedberg Campus

Max-von-Laue-Straße 15, 60438 Frankfurt am Main (Germany)

Prof. Dr. H. Ovaa, Dr. G. J. van der Heden van Noort

Department of Chemical Immunology, Leiden University Medical

Centre

Einthovenweg 20, 2333 ZC, Leiden (The Netherlands)

E-mail: gvanderheden@lumc.nl

Dr. H. A. V. Kistemaker

Current address: ProQR Therapeutics

Leiden (The Netherlands)

(9) Supporting information and the ORCID identification number(s) for the author(s) of this article can be found under:

https://doi.org/10.1002/anie.201710527. linkage to the protein. ${ }^{[1]}$ Mono-ADP-ribosylation is not only a PTM effected by bacterial toxins and the starting point for poly-ADP-ribosylation but also a regulatory modification in its own right. Mono-ADP-ribosylation is reported to take place on a variety of amino acid side chains, including arginine (Figure 1), glutamic acid, aspartic acid, asparagine, and

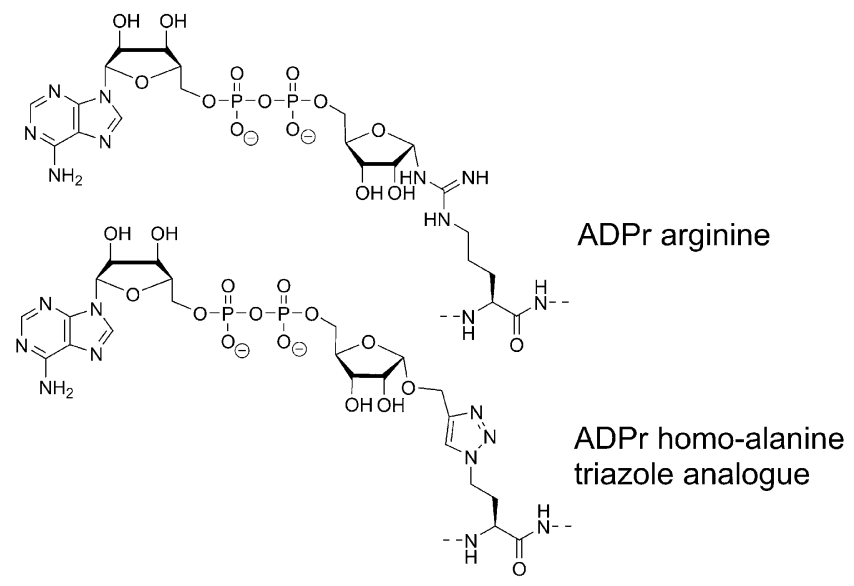

Figure 1. Structure of $\alpha$-linked ADPr arginine and ADPr triazole analogue linkages.

cysteine, but recently it was pointed out that serine might be the main point of attachment for ADP-ribosylation. ${ }^{[2]}$ Research in the field of protein ADP-ribosylation benefits greatly from ADP-ribosylated molecular tools. One way to obtain such tools in sufficient quantities is through chemical synthesis. Methods towards naturally occurring mono-ADPribosylated oligopeptides, ADPr oligomers, and NAD ${ }^{+}$-analogues have been reported and employed in studying ADPribosyl hydrolase affinity, ${ }^{[3]}$ inhibition of ADP-ribosylating toxins, finding substrate proteins for poly ADPr polymerases, ${ }^{[4]}$ and determining the structure of poly ADPribose glycohydrolases. ${ }^{[5]}$ Such ADPr peptides and related substances are valuable for the interrogation of the complex biology that underlies this PTM ${ }^{[6]}$ In the chemical synthesis of peptides and proteins, most commonly an acidic step to remove protective groups is employed. Such conditions, however, may cause either epimerization at the anomeric center of ribose or complete loss of the ADPr-moiety. Mild alkaline conditions, carry the risk of degradation of the $\beta$ substituted amino acids and are clearly incompatible with the esters of ADP-ribosylated Glu and ADP-ribosylated Asp. The reported syntheses of ADPr amino acids and peptides so 
far have been carefully tuned to minimize those risks and incorporation of ADPr amino acids asks for a modified protective group strategy in most cases. ${ }^{[6 a, 7]}$ To prevent the need for highly specialized methods to prepare these amino acid-ribose conjugates we propose a general strategy that would allow a post-synthetic introduction of the ADPr moiety to a peptide or protein of interest.

We selected the ADPr triazole analogues as a relevant replacement for ADPr amino acids in peptides (Figure 1). Oligopeptides with an azide incorporated can be obtained by standard solid phase peptide synthesis (SPPS) using an azidoalanine or azido-homoalanine building block at the site of the modification. After conventional synthesis and a coppercatalyzed azide-alkyne cycloaddition (CuAAC) of the obtained azido functionalized oligopeptide with a suitable propargylated ADPr building block (ADPr-pr, 7 in Scheme 1) results in the installation of the ADPr triazole functionality.

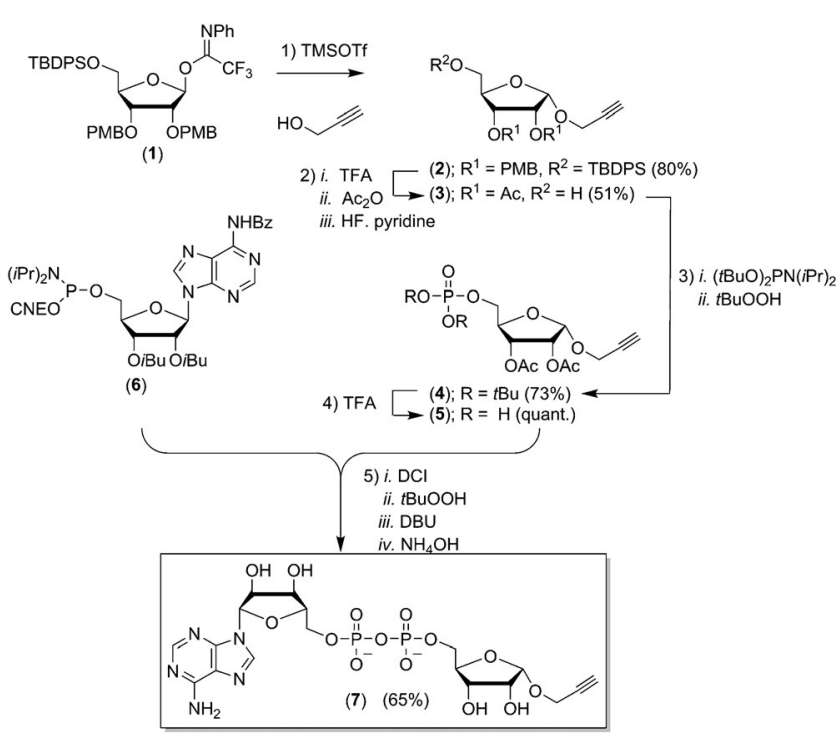

Scheme 1. Synthesis of ADPr-propargyl 7.

The synthesis of ADPr-pr $\mathbf{7}$ starts with the condensation of propargyl alcohol with imidate ribose donor $\mathbf{1}^{\text {[7a] }}$ (Scheme 1) to yield an anomeric mixture $(\alpha: \beta, 71: 29)$. In contrast to two previously reported syntheses that show the preparation of either $\beta$ - $O$-alkyne or $\beta$-azide containing ADPr analogues, we were able to isolate the biologically relevant $\alpha$-anomer. ${ }^{[5,8]}$ Protective-group exchange allows alkaline deprotection in the final stage of the synthesis instead of acid treatment, preventing possible degradation of the ADPr-pr moiety. Phosphitylation of the primary alcohol and subsequent oxidation furnishes crucial phosphotriester intermediate 4. Removal of the $t \mathrm{Bu}$ groups set the stage for the installation of the pyrophosphate moiety using our previously reported procedure. ${ }^{[9]}$ Reaction of phosphomonoester $\mathbf{5}$ with suitably protected adenosine phosphoramidite $\mathbf{6}^{[10]}$ using dicyanoimidazole (DCI) as activator and oxidation of the intermediate $\mathrm{P}^{\mathrm{III}}-\mathrm{P}^{\mathrm{V}}$ species was followed by a two-step alkaline deprotection procedure. Purification using size exclusion chromatography gave access to ADPr-pr 7 in a quantity of $100 \mathrm{mg}$.
Formation of the pyrophosphate and subsequent deprotection proceeded in an overall isolated yield of $65 \%$, which compares favorably to other approaches. ${ }^{[6,8,11]}$

To assess the viability of ADPr-pr building block 7 in the projected cycloaddition, we prepared three peptides derived from mono-ADP-ribosylated proteins; namely Histone $\mathrm{H} 2 \mathrm{~B}$ (2-14) (see Scheme 2, compound 8), RhoA (36-46) (Scheme 2, compound 9) and human neutrophil defensin 1;
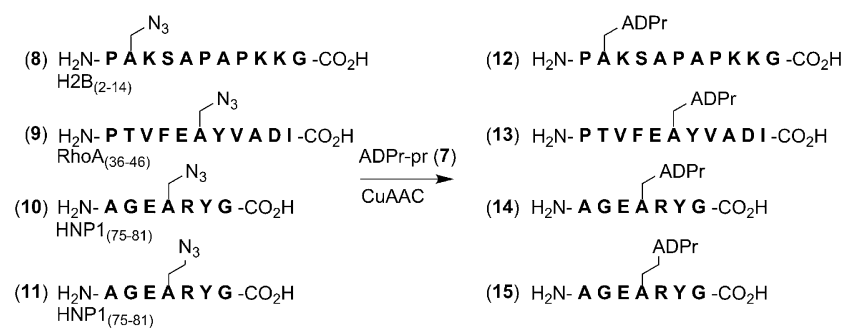

Scheme 2. CuAAC reaction towards ADPr peptides.

HNP1 (75-81) (see Scheme 2, compound 10). In the selected peptides, we substituted Gln3, Asn50, and Arg78, respectively, for $\beta$-azidoalanine to allow conjugation by coppercatalyzed cycloaddition. After completion of the SPPS, the immobilized oligopeptides were treated with a cleavage mixture consisting of $90.5 \%$ trifluoroacetic acid (TFA), $5 \%$ water, $2.5 \%$ phenol and $2 \%$ triisopropylsilane to globally remove the protective groups and cleave the peptide from the resin. RP-HPLC purification yielded target peptides 8-10 that were used in the CuAAC reaction with ADPr-pr 7 in $20 \mathrm{~mm}$ tris(hydroxymethyl)aminomethane $/ 150 \mathrm{~mm} \mathrm{NaCl}$ buffer at $\mathrm{pH} 7.6$ under the agency of $10 \mathrm{~mm} \mathrm{CuSO}_{4}, 60 \mathrm{~mm}$ sodium ascorbate, and $10 \mathrm{~mm}$ tris triazole ligand. ${ }^{[12]}$ Since these peptides and ADPr-pr 7 dissolved readily in this buffer, the click reaction proceeded efficiently and quickly (within minutes to one hour), in contrast to a previous study. ${ }^{[8]}$ To mimic the length of the Arg-ADPr linkage more closely, we prepared HNP1-peptide $\mathbf{1 1}$ in which the Arg was replaced not with $\beta$-azidoalanine but with azido-homoalanine. Again CuAAC to ADPr-pr proceeded uneventfully, yielding 15.

Having established an efficient procedure to prepare ADPr oligopeptides, we were eager to see whether our method could be expanded in preparation of ADPr proteins. Ubiquitin (Ub), a 76 amino acid residue long post-translational modifier itself, has recently been found to be modified with ADPr on different positions. This cross-talk between ADP-ribosylation and ubiquitination is reported to have a regulatory effect on the DNA repair mechanism, where low levels of NAD lead to ubiquitination of histone protein $\mathrm{H} 4$, but high levels of NAD lead to ADP-ribosylation of Gly76; the C-terminus of Ub. ${ }^{[13]}$ Other studies show that Arg42 of Ub is ADP-ribosylated by a family of effector proteins originating from Legionella pneumophila, the pathogen causing Legionnaires' disease. ${ }^{[14]}$ These SidE effectors are the first reported class of enzymes that are able to ubiquitinate target proteins independent of the normally employed enzymatic cascade of E1, E2, and E3 enzymes, utilizing Ub-ADPr as crucial intermediate. Using their unique properties, SidE 
proteins can hijack the host cells Ub pool and use it to its own advantage. In analogy to peptide $\mathbf{1 1}$, we prepared Ub mutant $\mathbf{1 6}$ in which Arg42 is replaced by azido-homoalanine using our previously published linear SPPS approach. ${ }^{[15]}$ Gly76 modified Ub 17 was prepared by first synthesizing $\mathrm{Ub}_{75}$ on trityl resin followed by treatment with mild acid $(20 \%$ hexafluoroisopropanol in dichloromethane). In this step the peptide was liberated from the solid support while leaving all sidechain protecting groups in place. Activation of the free Cterminal carboxylic acid and coupling of 3-azido-1-propanamine followed by strong acid treatment and RP-HPLC purification yielded azide modified Ub 17. Copper-catalyzed click reaction with ADPr-pr building block $\mathbf{7}$ followed by dialysis to remove traces of excess ADPr-pr and click reagents followed by size exclusion chromatography gave easy access to ADP-ribosylated ubiquitin analogues $\mathbf{1 8}$ and $\mathbf{1 9}$, respectively (Scheme 3).

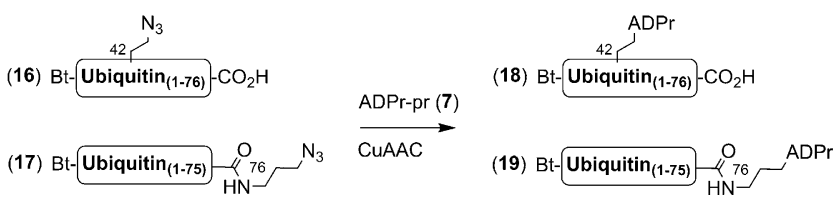

Scheme 3. CuAAC reaction towards Ub-ADPr conjugates.

Of note is that this procedure does not require the use of RP-HPLC purification after introduction of the ADPr moiety. To assess whether the artificial triazole linkage is tolerated and this method indeed results in useful ADPrprotein analogues, we compared Ub-ADPr 18 to Arg42 UbADPr from natural sources (Ub-ADPr wt). Both Ub-ADPr 18 and Ub-ADPr wt were efficiently recognized by an ADPrantibody in western blot (Figure $2 \mathrm{~A}$, lower panel), a first indication that the triazole analogue does not differ too far from its natural counterpart. One of the properties of Legionella effector SdeA is its auto-ubiquitination behavior, an effect that is not fully understood so far, but is reported for all four SidE family members. We compared the ability of recombinant SdeA to use $\mathbf{1 8}$ in an auto-ubiquitination assay and found indeed that SdeA is modified with $\mathrm{Ub}$ multiple times (see Figure 2A,B). Although at a reduced rate compared to Ub-ADPr wt, artificial $\mathbf{1 8}$ was processed by SdeA and significant auto-ubiquitination takes place. A control
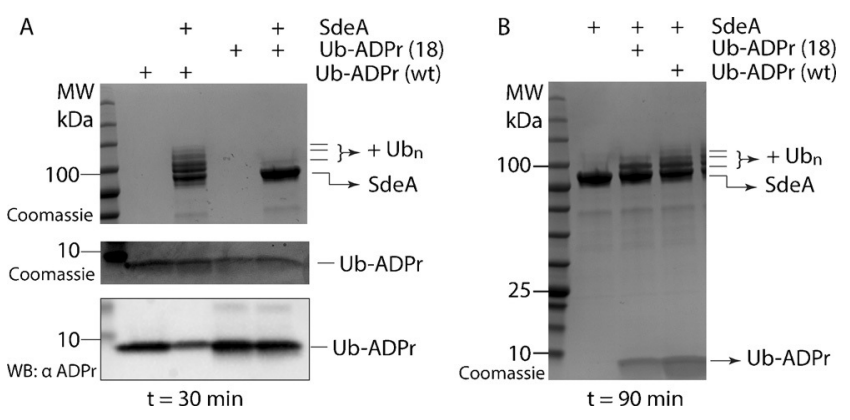

Figure 2. Comparison of Arg42 Ub-ADPr wt and Ub-ADPr analogue 18 processing by SdeA at A) $30 \mathrm{~min}$ and B) $90 \mathrm{~min}$. experiment using non-ADPribosylated wild type Ub shows no auto-ubiquitination of SdeA (Figure 1; Supporting Information). These results further confirm that Ub-ADPr conjugate 18 functions similar to Ub-ADPr wt.

In conclusion, we have presented the design and synthesis of propargylated ADP-ribose building block (7) suitable to take part in an efficient cycloaddition with oligopeptides and proteins having an azide at a predetermined position. In this way oligopeptides and proteins carrying an analogue of the mono ADP-ribose post-translational modification are made available. Four ADPr-oligopeptide conjugates derived from known ADP-ribosylated proteins were prepared efficiently. Furthermore, two analogues of ADPr ubiquitin, shown to play a role in Legionnaires' disease and DNA repair, were prepared using the same copper-catalyzed chemistry. The effectiveness of these reactions and subsequent purifications provides an easy entry to this interesting class of posttranslational modified proteins. Triazole-containing UbADPr 18 was shown to be recognized in western blot and accepted by SdeA in an auto-ubiquitination assay, indicating that this method provides a useful platform for the biological interrogation of ADPr biology.

\section{Acknowledgements}

We like to thank Dris el Atmioui and Cami Talavera Ormeňo for SPPS. This work was supported by a NWO VENI-grant to G.J.v.d.H.v.N. and by an NWO ECHO-grant to H.A.V.K.

\section{Conflict of interest}

The authors declare no conflict of interest.

Keywords: ADP-ribosylation - click chemistry . post-translational modification - protein modification . ubiquitination

How to cite: Angew. Chem. Int. Ed. 2018, 57, 1659-1662 Angew. Chem. 2018, 130, 1675-1678

[1] a) K. Ueda, O. Hayaishi, J. Oka, H. Komura, K. Nakanishi in ADP-Ribosylation of Proteins (Eds.: F. R. Althaus, H. Hilz, S. Shall), Springer Berlin Heidelberg, Berlin, 1985, pp. 159-166; b) G.-C. Zhou, S. L. Parikh, P. C. Tyler, G. B. Evans, R. H. Furneaux, O. V. Zubkova, P. A. Benjes, V. L. Schramm, J. Am. Chem. Soc. 2004, 126, 5690-5698.

[2] a) Q. Liu, B. I. Florea, D. V. Filippov, Cell Chem. Biol. 2017, 24, 431-432; b) J. J. Bonfiglio, P. Fontana, Q. Zhang, T. Colby, I. Gibbs-Seymour, I. Atanassov, E. Bartlett, R. Zaja, I. Ahel, I. Matic, Mol. Cell 2017, 65, 932-940.e936.

[3] a) H. A. V. Kistemaker, A. P. Nardozza, H. S. Overkleeft, G. A. van der Marel, A. G. Ladurner, D. V. Filippov, Angew. Chem. Int. Ed. 2016, 55, 10634-10638; Angew. Chem. 2016, 128, $10792-$ 10796; b) R. L. McPherson, R. Abraham, E. Sreekumar, S.-E. Ong, S.-J. Cheng, V. K. Baxter, H. A. V. Kistemaker, D. V. Filippov, D. E. Griffin, A. K. L. Leung, Proc. Natl. Acad. Sci. USA 2017, 114, 1666-1671.

[4] H. Jiang, J. H. Kim, K. M. Frizzell, W. L. Kraus, H. Lin, J. Am. Chem. Soc. 2010, 132, $9363-9372$. 
[5] M. J. Lambrecht, M. Brichacek, E. Barkauskaite, A. Ariza, I Ahel, P. J. Hergenrother, J. Am. Chem. Soc. 2015, 137, 3558 3564.

[6] a) G. J. van der Heden van Noort, M. G. van der Horst, H. S. Overkleeft, G. A. van der Marel, D. V. Filippov, J. Am. Chem. Soc. 2010, 132, 5236-5240; b) P. M. Moyle, T. W. Muir, J. Am. Chem. Soc. 2010, 132, 15878-15880.

[7] a) H. A. V. Kistemaker, G. J. van der Heden van Noort, H. S Overkleeft, G. A. van der Marel, D. V. Filippov, Org. Lett. 2013 15, 2306-2309; b) G. Speciale, A. Bernardi, F. Nisic, Molecules 2013, 18, 8779 .

[8] L. Li, Q. Li, S. Ding, P. Xin, Y. Zhang, S. Huang, G. Zhang, Molecules 2017, 22, 1346.

[9] a) H. A. V. Kistemaker, L. N. Lameijer, N. J. Meeuwenoord, H. S. Overkleeft, G. A. van der Marel, D. V. Filippov, Angew. Chem. Int. Ed. 2015, 54, 4915-4918; Angew. Chem. 2015, 127, $4997-5000$; b) H. Gold, P. van Delft, N. Meeuwenoord, J. D. C. Codée, D. V. Filippov, G. Eggink, H. S. Overkleeft, G. A van der Marel, J. Org. Chem. 2008, 73, 9458-9460.

[10] G. J. van der Heden van Noort, H. S. Overkleeft, G. A. van der Marel, D. V. Filippov, J. Org. Chem. 2010, 75, 5733-5736.
[11] G. J. van der Heden van Noort, C. P. Verhagen, M. G. van der Horst, H. S. Overkleeft, G. A. van der Marel, D. V. Filippov, Org. Lett. 2008, 10, $4461-4464$.

[12] Z. Zhou, C. J. Fahrni, J. Am. Chem. Soc. 2004, 126, 8862-8863.

[13] C.-S. Yang, K. Jividen, A. Spencer, N. Dworak, L. Ni, L. T. Oostdyk, M. Chatterjee, B. Kuśmider, B. Reon, M. Parlak, V. Gorbunova, T. Abbas, E. Jeffery, N. E. Sherman, B. M. Paschal, Mol. Cell 2017, 66, 503-516.e505.

[14] a) S. Bhogaraju, S. Kalayil, Y. Liu, F. Bonn, T. Colby, I. Matic, I. Dikic, Cell 2016, 167, 1636-1649.e1613; b) K. Puvar, Y. Zhou, J. Qiu, Z.-Q. Luo, M. J. Wirth, C. Das, Biochemistry 2017, 56, 4762-4766; c) J. Qiu, M. J. Sheedlo, K. Yu, Y. Tan, E. S. Nakayasu, C. Das, X. Liu, Z.-Q. Luo, Nature 2016, 533, 120-124.

[15] F. El Oualid, R. Merkx, R. Ekkebus, D. S. Hameed, J. J. Smit, A. de Jong, H. Hilkmann, T. K. Sixma, H. Ovaa, Angew. Chem. Int. Ed. 2010, 49, 10149-10153; Angew. Chem. 2010, 122, 10347 10351.

Manuscript received: October 12, 2017

Revised manuscript received: November 24, 2017

Accepted manuscript online: December 7, 2017

Version of record online: January 8, 2018 